\title{
Vol. 70, No. 14
}

In the report "Provisional Mortality Data - United States, 2020," on page 520, the last sentence in the "What is added by this report?" paragraph of the Summary box should have read, "COVID-19 was the third leading cause of death, and the COVID-19 death rate was highest among non-Hispanic American Indian or Alaska Native persons." 